\title{
Efficiency of mechanical activation of inspiratory muscles in COPD using sample entropy
}

To the Editor:

Respiratory muscle dysfunction is a common problem in patients with chronic obstructive pulmonary disease (COPD) and has mostly been related to pulmonary hyperinflation $[1,2]$. Associated diaphragm shortening and deleterious changes in the muscle force-length relationship cause a reduction in the muscles' capacity to generate pressure, placing them at a mechanical disadvantage $[1,3]$. Specifically, both inspiratory muscle strength and mechanical efficiency may be reduced in COPD patients [1,4-6], although, at isovolume, the contractile strength of the diaphragm in COPD is preserved or may even be improved in some cases [7]. The ratio between transdiaphragmatic pressure and electrical diaphragm activity has been used as a measure of respiratory muscle efficiency $[8,9]$. However, in clinical practice, it is complex to measure this parameter directly, as invasive measures are required and these are uncomfortable for patients [4].

During contraction, respiratory muscle fibres vibrate laterally [10]. These vibrations are related to the mechanical activation of these muscles and can be noninvasively recorded through accelerometers positioned on the surface of the skin, proximal to the muscles: this is called respiratory muscle mechanomyogram (MMG) [11-13]. The analysis of the mechanical activation of inspiratory muscles through the MMG might be a useful alternative approach for assessing respiratory muscles function in patients with COPD [13, 14]. MMG reflects the mechanical counterpart of the neural activity measured by electromyography. Respiratory muscle MMG provide some advantages over surface diaphragmatic electromyography with regards to simplicity of use. First of all, MMG recording is easy and simple to implement; MMG is acquired using a small accelerometer attached to the skin surface, whereas electromyography typically uses three electrodes. Secondly, as it is a mechanical signal, MMG is not susceptible to bioelectrical interference. Furthermore, the signal to noise ratio of $\mathrm{MMG}$ is typically higher than that of the electromyography, requiring less amplification and electrical shielding. In addition, the MMG recording does not require skin preparation and it is not influenced by changes in the skin impedance.

The aim of the present study was to noninvasively evaluate the mechanical activation of inspiratory muscles and its efficiency (EMMG) during tidal volume breathing in patients with severe-to-very severe COPD. With this in mind, we investigated the maximal inspiratory pressure ( $P$ Imax) and respiratory muscle MMG acquired under both quiet breathing (QB) and maximal voluntary ventilation (MVV) conditions during an incremental respiratory flow protocol.

A group of 10 male COPD patients participated in the study. Five of the patients had severe COPD (S-COPD) with a mean \pm SD for age of $72.8 \pm 4.1$ years, body mass index (BMI) of $23.7 \pm 4.1 \mathrm{~kg} \cdot \mathrm{m}^{-2}$, and forced expiratory volume in $1 \mathrm{~s}$ (FEV1) of $40.0 \pm 6.5 \%$ pred). The remaining five had very severe COPD (VS-COPD) and aged $63.7 \pm 8.2$ years, with a BMI of $21.7 \pm 3.0 \mathrm{~kg} \cdot \mathrm{m}^{-2}$ and an FEV1 of $20.5 \pm 3.0 \%$ pred). The severity of the disease was classified in accordance with the American Thoracic Society/European Respiratory Society criteria [15]. A higher static hyperinflation and greater trapping of air was observed in VS-COPD (mean \pm SD residual volume (RV) $284 \pm 66 \%$ pred versus $191 \pm 6 \%$ pred in S-COPD; RV/total lung capacity $\%$ in VS-COPD was $77 \pm 3$ versus $68 \pm 5$ in S-COPD). The study was conducted with the written consent of patients, the approval of the Ethics Committee of Hospital del Mar (Barcelona, Spain) and in conformity with the declaration of Helsinki for studies in humans.

PImax and MMG recordings were made with patients sitting quietly and breathing through a mouthpiece and a tube, while wearing a nose clip. An experienced physician instructed the patients to regulate their respiration, progressively and controllably increasing the rhythm and intensity of their breathing from QB to reach their MVV. Subsequently, they progressively decreased the rhythm and intensity of their breathing until they again reached the QB level. Each patient performed this respiratory protocol three times. The mean respiratory frequency during QB periods ranged from 14 to 25 breaths $\min ^{-1}$ (mean \pm SD were $18.7 \pm 3.3$ and $18.0 \pm 4.3$ for S-COPD and VS-COPD, respectively), whereas during MVV periods the mean respiratory frequency ranged from 32 to 70 breaths. $\mathrm{min}^{-1}$ (mean \pm SD were $53.6 \pm 16.0$ and 42.6 \pm 8.0 for VS-COPD and S-COPD, respectively).

The PImax was recorded with a pressure transducer (Digima Premo 355, Special Instruments, Noerdlingen, Germany) placed in the tube through which the patients breathed. Two MMG were simultaneously recorded with two capacitive accelerometers (8312B2; Kistler, Winterthur, Switzerland). Accelerometers 

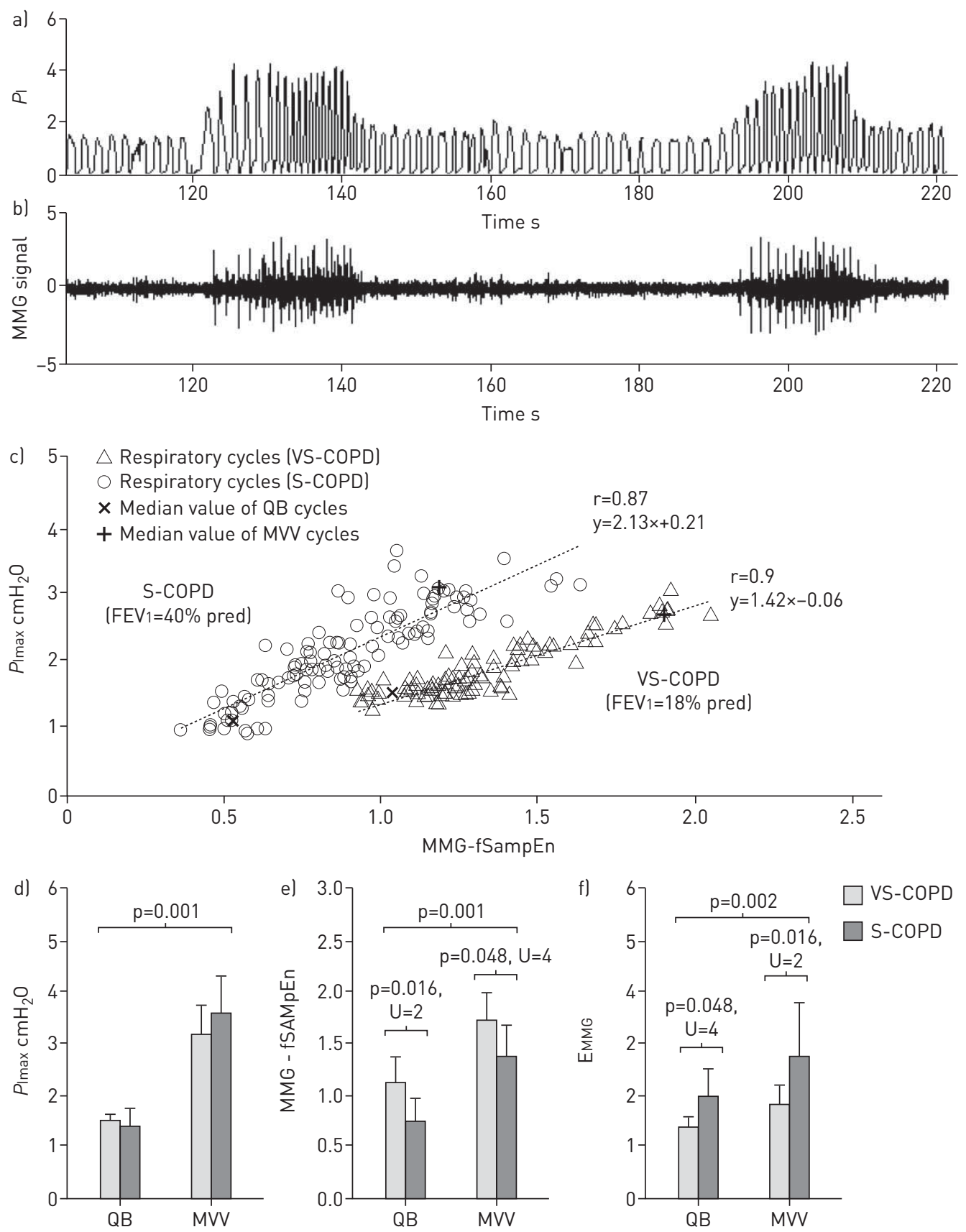

FIGURE 1 Representative traces during the incremental respiratory flow protocol of a) inspiratory pressure $(P \mathrm{I})$ and $\mathrm{b}$ ) the average respiratory muscle mechanomyogram (MMG) recordings. c) Scatter plot of inspiratory muscle effort estimated through maximal PImax and mechanical activation of inspiratory muscles estimated with fixed sample entropy (fSampEn) in two representative patients: severe (S) chronic obstructive pulmonary disease (COPD) and very severe (VS)-COPD. Quiet breathing (QB) cycles were considered the only respiratory cycles before the first incremental flow manoeuvre. The maximum voluntary ventilation (MVV) cycles were selected as $20 \%$ of the cycles for the incremental flow respiratory test with the highest PImax. Mean \pm SD values for d) PImax, e) MMG-fSampEn, and f) efficiency of mechanical activation of inspiratory muscle estimated by index EMMG defined as the ratio between PImax and MMG-fSampEn. FEV1: forced expiratory volume in $1 \mathrm{~s}$.

were placed on the chest surface, between the seventh and eighth intercostal spaces at the left and right anterior axillary lines, respectively. This position is proximal to the zone of apposition of the diaphragm muscle. Thus, this muscle will probably be the main contributor to the MMG signals, although intercostal muscles of the lower chest wall may also contribute. Left and right MMG recordings were averaged to obtain more robust information about the mechanical activation of inspiratory muscles contribution of both hemidiaphragms. As an example, figure 1a) and b) show the inspiratory pressure $P$ I and the average of the MMG recordings, respectively, of one S-COPD patient. 
The PImax obtained in each respiratory cycle was considered as a gold standard reference for quantification of respiratory muscle effort during tidal volume. The fixed sample entropy (fSampEn) is a novel index used to estimate the respiratory muscle effort from MMG recordings [12]. The MMG-fSampEn was calculated for each inspiratory cycle of the averaged MMG. Advantages of fSampEn index over classical methods can be found in $[12,16]$.

The EMMG was computed as the ratio between the effort produced by inspiratory muscles at a specific level of tidal breathing (assessed by the PImax) and the mechanical activation of inspiratory muscles achieved to produce this effort (estimated by MMG-fSampEn). A high value of EMMG indicates that the patient is able to generate the same PImax with lower levels of MMG.

PImax, MMG-fSampEn and EMMG values were compared between S-COPD and VS-COPD groups for the same tidal volume manoeuvres (QB and MVV) using the one-sided Mann-Whitney U test. Results were also compared between QB and MVV (for all COPD patients) using the one-sided Wilcoxon signed rank test.

A strong linear relation between PImax and the MMG-fSampEn was found (figure 1c). Pearson's correlation coefficient was computed considering all the respiratory cycles for each patient individually (mean $\pm \mathrm{SD}$ were $0.80 \pm 0.14$ for VS-COPD and $0.86 \pm 0.11$ for S-COPD). Increased inspiratory muscle effort leads to an increase in mean values of PImax, although this occurs in a different way as a function of the severity of COPD patients. On the one hand, for QB there were slight differences in PImax between S-COPD and VS-COPD (figure 1d), showing no relationship with lung function. However, under MVV conditions the PImax decreased with increasing severity of patients (figure 1d). In contrast, VS-COPD patients achieved higher levels of MMG-fSampEn than S-COPD patients in both QB $(p=0.016, \mathrm{U}=2)$ and MVV $(\mathrm{p}=0.048$, $\mathrm{U}=4$ ) conditions (figure 1e). Overall, these results suggest that, due to the greater degree of airflow obstruction, individuals with VS-COPD need greater mechanical activation of inspiratory muscles to achieve similar PImax at MVV to those with S-COPD.

The EMMG increased significantly $(\mathrm{p}=0.002)$ with the inspiratory effort from QB to MVV. Furthermore, for both QB and MVV, the EMMG was higher in S-COPD than VS-COPD patients (figure 1f). Significant differences were observed for both QB ( $\mathrm{p}=0.048, \mathrm{U}=4)$ and MVV $(\mathrm{p}=0.016, \mathrm{U}=2)$.

The use of PImax in the present study can be controversial. On the one hand, this variable represents the overall mechanical output of inspiratory muscles and not only that of the diaphragm. However, the same accounts for the MMG signal that is predominantly produced by the diaphragm but also integrates a contribution from muscles of the lower chest wall. This point actually reflects physiological and clinical conditions, since it is very well known that when there is an increase in respiratory load, chest wall muscles progressively contribute to the ventilatory effort $[17,18]$. This would be of special interest in the MVV situation. In addition, mouth pressure can be non-invasively recorded and, as previously mentioned, actually reflect the resulting mechanical outcome of all inspiratory muscles, both factors with clear advantages in clinical situations.

To our knowledge, the current study shows, for the first time, a direct comparison between the mechanical activation of the inspiratory muscles, estimated through the amplitude of the noninvasive MMG, and the inspiratory effort at tidal volume. Our results indicate that the MMG of patients with severe-to-very severe COPD is closely related to the inspiratory effort, according to the PImax. Furthermore, we have shown that the MMG is higher in more severe patients. In other words, the greater the degree of airflow obstruction the greater mechanical activation of inspiratory muscle requires. Although the results should be interpreted with caution, our observations indicate that the EMMG, calculated as the ratio between PImax and estimation of mechanical activation of inspiratory muscles, decreases with increasing COPD severity. Therefore, patients with very severe COPD would show a worse efficiency of inspiratory muscles.

In conclusion, the data support the use of MMG as a noninvasive alternative to quantification of the efficiency of the mechanical activation of inspiratory muscles in patients with COPD, and, potentially, could be a useful tool to evaluate disease progression and could provide valuable information on the effect of treatments.

@ERSpublications

Respiratory muscle mechanomyogram exhibits significant differences between severe and very severe COPD patients http://ow.ly/SdV6Y

Leonardo Sarlabous ${ }^{1,2}$, Abel Torres ${ }^{1,2,3}$, José A. Fiz ${ }^{1,2,4}$, Joaquim Gea ${ }^{5}$, Juana M. Martínez-Llorens ${ }^{5}$ and Raimon Jané ${ }^{1,2,3}$ ${ }^{1}$ Institut de Bioenginyeria de Catalunya (IBEC), Barcelona, Spain. ${ }^{2}$ CIBER de Bioingeniería, Biomateriales y Nanomedicina (CIBER-BBN), Barcelona, Spain. ${ }^{3}$ Dept ESAII, Universitat Politècnica de Catalunya, Barcelona, Spain. ${ }^{4}$ Dept of Pulmonology, Germans Trias i Pujol University Hospital, Badalona, Spain. ${ }^{5}$ Respiratory Medicine Dept, Hospital del Mar IMIM. DCEXS, UPF. CIBERES, ISCiii, Barcelona, Spain.

Correspondence: Abel Torres, Cebrián, Institute for Bioengineering of Catalonia (IBEC), C. Baldiri Reixac 4, 08028, Barcelona, Spain. E-mail: atorres@ibecbarcelona.eu 
Support statement: This research was funded in part from a grant by Secretaria d'Universitats i Recerca del Departament d'Economia i Coneixement de la Generalitat de Catalunya, grant number GRC 2014 SGR 1569. Funding information for this article has been deposited with FundRef.

Conflict of interest: Disclosures can be found alongside the online version of this article at erj.ersjournals.com

\section{References}

1 Rochester D, Braun N. Determinants of maximal inspiratory pressure in chronic obstructive pulmonary disease. Am Rev Respir Dis 1985; 132: 42-47.

2 Ottenheijm CAC, Heunks LMA, Sieck GC, et al. Diaphragm dysfunction in chronic obstructive pulmonary disease. Am J Respir Crit Care Med 2005; 172: 200-205.

3 McKenzie DK, Butler JE, Gandevia SC. Respiratory muscle function and activation in chronic obstructive pulmonary disease. J Appl Physiol 2009; 107: 621-629.

4 Celli BR, MacNee W, Agusti A, et al. Standards for the diagnosis and treatment of patients with COPD: a summary of the ATS/ERS position paper. Eur Respir J 2004; 23: 932-946.

5 Finucane KE, Singh B. Human diaphragm efficiency estimated as power output relative to activation increases with hypercapnic hyperpnea. J Appl Physiol 2009; 107: 1397-1405.

6 Gosselink RA, Wagenaar RC, Rijswijk H, et al. Diaphragmatic breathing reduces efficiency of breathing in patients with chronic obstructive pulmonary disease. Am J Respir Crit Care Med 1995; 151: 1136-1142.

7 Similowski T, Yan S, Gauthier AP, et al. Contractile properties of the human diaphragm during chronic hyperinflation. N Engl J Med 1991; 325: 917-923.

8 Beck J, Sinderby C, Lindström L, et al. Effects of lung volume on diaphragm EMG signal strength during voluntary contractions. J Appl Physiol 1998; 85: 1123-1134.

9 Liu L, Liu H, Yang Y, et al. Neuroventilatory efficiency and extubation readiness in critically ill patients. Crit Care; 2012; 16: R143.

10 Petitjean M, Bellemare F. Phonomyogram of the diaphragm during unilateral and bilateral phrenic nerve stimulation and changes with fatigue. Muscle Nerve 1994; 17: 1201-1209.

11 Sarlabous L, Torres A, Fiz JA, et al. Index for estimation of muscle force from mechanomyography based on the Lempel-Ziv algorithm. J Electromyogr Kinesiol 2013; 23: 548-557.

12 Sarlabous L, Torres A, Fiz JA, et al. Evidence towards improved estimation of respiratory muscle effort from diaphragm mechanomyographic signals with cardiac vibration interference using sample entropy with fixed tolerance values. PLoS One 2014; 9: e88902.

13 Torres A, Fiz JA, Sarlabous L, et al. Noninvasive evaluation of the respiratory muscular function by means of mechanomyographic signals of the diaphragm muscle in COPD patients. Eur Respir J 2010; 36: Suppl. 54, 503s.

14 American Thoracic Society/European Respiratory Society: ATS/ERS statement on respiratory muscle testing. Am J Respir Crit Care Med 2002; 166: 518-624.

15 Bakke PS, Rönmark E, Eagan T, et al. Recommendations for epidemiological studies on COPD. Eur Respir J 2011; 38: 1261-1277.

16 Estrada L, Torres A, Sarlabous L, et al. Improvement in neural respiratory drive estimation from diaphragm electromyographic signals using fixed sample entropy. IEEE J Biomed Health Inform 2015 [in press; DOI: 10.1109/ JBHI.2015.2398934].

17 Duiverman ML, Van Eykern LA, Vennik PW, et al. Reproducibility and responsiveness of a noninvasive EMG technique of the respiratory muscles in COPD patients and in healthy subjects. J Appl Physiol 2004; 96: 1723-1729.

18 De Troyer A, Kirkwood PA, Wilson TA. Respiratory action of the intercostal muscles. Physiol Rev 2005; 85: $717-756$.

\section{Poor standardisation of plethysmographic specific airways resistance measurement despite widespread use}

To the Editor:

Plethysmographic specific airways resistance $\left(s R_{\mathrm{aw}}\right)$ has remained the most recognised measure of airway resistance for almost 60 years [1]. Studies in both adults and children (including preschoolers) suggest clinical utility across important obstructive lung diseases [2-5]. Measurement protocols based on rapid panting or tidal breathing exist, and are incorporated into many current commercial plethysmographs. Tidal breathing measurement offers feasibility across a wide age range, and a relatively stable index with 